\title{
CARACTERIZACIÓN DE LA FUNCIÓN DE VALOR EMPLEADA EN LAS DECISIONES AMBIENTALES POR LAS GRANDES OR- GANIZACIONES: ESTUDIO DE LOS GRANDES PROYECTOS HIDROELÉCTRICOS EN COLOMBIA*
}

\author{
RODOLFO GARCÍA SIERRA** \& ÁLVARO ZERDA SARMIENTO**** \\ UNIVERSIDAD NACIONAL DE COLOMBIA
}

Recibido/ Received/ Recebido: 09/05/2016 - Aceptado/ Accepted / Aprovado: 10/10/2016

\begin{abstract}
Resumen
Esta investigación ofrece una explicación sobre una de las fuentes generadoras de los altos niveles de conflictividad, actualmente predecibles entre las comunidades locales y la construcción de megaproyectos hidroeléctricos en Colombia, a través de una mirada endógena de las grandes organizaciones, específicamente en su proceso de toma de decisiones ambientales basado en juicio de expertos. En este caso el análisis se aplica a la construcción de megaproyectos hidroeléctricos (>350 MW) en Colombia durante el período 2010 al 2020. La investigación emplea la teoría prospectiva acumulativa (Cumulative Prospect Theory - CPT). Se inicia determinando el uso de la heurística de disponibilidad y su cuantificación, a partir de los criterios de facilidad de recordación y tiempo de deliberación de las respuestas. Paso siguiente, se caracteriza la función de preferencias de los expertos, determinando las dimensiones en las que se presentan zonas de aptitudes de ganancia (altas y bajas) y zonas de aptitudes de pérdidas (bajas y altas). Su aplicación a las organizaciones según las simulaciones realizadas, permite mejorar la influencia de las comunidades locales en las decisiones ambientales, teniendo presente los resultados sobre las aptitudes de pérdidas que han experimentado los tomadores de decisiones, ante peticiones externas en las dimensiones tecnológicas y ambientales.
\end{abstract}

Palabras clave: Teoría prospectiva acumulativa; Juicio experto; Toma de decisiones; Megaproyectos; Hidroeléctricas.

El artículo es producto de un resultado de la investigación realizada en el trabajo doctoral: Toma de decisiones por grandes organizaciones en condiciones de Incertidumbre: Estudio de las grandes hidroeléctricas en Colombia 2010-2020. Finalizado en Diciembre 2016. Universidad Nacional de Colombia, para optar al título de Doctor en Ingeniería - Industria y Organizaciones.

* PhD, M.Sc., EE. Profesor Ocasional Universidad Nacional de Colombia. Integrante Grupo Investigación: Observatorio de Economía Internacional rgarciasi@unal.edu.co

"** PhD, M.Sc., Eco. Profesor Asociado Universidad Nacional de Colombia. Integrante Grupo Investigación: Observatorio de Economía Internacional azerdas@unal.edu.co 


\title{
FUNCTION OF VALUE CHARACTERIZATION USED BY LARGE ORGANIZATIONS IN ENVIRONMENTAL DECISIONS: A STUDY OF THE MAJOR HYDROELECTRIC PROJECTS IN COLOMBIA
}

\begin{abstract}
This research offers an explanation of one of the generating sources of high levels of conflict, currently predictable among local communities and the construction of hydroelectric megaprojects in Colombia, through an endogenous view of large organizations, specifically in their process of taking of environmental decisions based on expert judgment. In this case the analysis is applied to the construction of hydroelectric megaprojects (> $350 \mathrm{MW}$ ) in Colombia during the period 2010 to 2020. The research uses the cumulative prospective theory (CPT). It begins by determining the use of the heuristic of availability and its quantification, based on the criteria of ease of recall and answers deliberation time. Next step, the function of preferences of the experts is characterized, determining the dimensions in which there are zones of aptitudes of gain (highs and lows) and zones of aptitudes of losses (lows and highs). Its application to organizations according to the simulations carried out, allows to improve the influence of local communities in environmental decisions, taking into account the results on the aptitudes of losses experienced by decision makers, in response to external requests in the technological and environmental dimensions.

Keywords: Cumulative prospective theory; Expert judgment; Decision making; Megaprojects; Hydroelectric.

\section{CARACTERIZAÇÃO DA FUNÇÃO DE VALOR EMPREGADA PELAS GRANDES ORGANIZAÇÕES NAS DECISÕES AMBIENTAIS: UM ESTUDO DOS GRANDES PROJETOS HIDROELÉTRICOS NA COLÔMBIA}

\begin{abstract}
Resumo
Esta pesquisa oferece uma explicação sobre uma das fontes geradoras de altos níveis de conflito, atualmente previsiveis, entre as comunidades locais e a construção de megaprojetos hidroelétricos na Colômbia, através de um olhar endógeno sobre as grandes organizações, especificamente em seu processo de toma de decisões ambientais, baseado em julgamento por parte de peritos. Neste caso a análise aplica-se à construção de megaprojetos hidroelétricos (>350 MW) na Colômbia durante o período de 2010 a 2020 . A pesquisa emprega a teoria prospectiva acumulativa (Cumulative Prospect Theory - CPT). Inicia-se determinando o uso da heurística de disponibilidade e sua quantificação, a partir dos critérios de facilidade de recordação e tempo de deliberação das respostas. Passo seguinte, caracteriza-se a função de preferências dos peritos, determinando as dimensões nas que se apresentam zonas de aptidões de ganho (altos e baixos) e zonas de aptidões de perdas (baixas e altas). Sua aplicação às organizações segundo as simulações realizadas, permite melhorar a influência das comunidades locais nas decisões ambientais, considerando os resultados sobre as aptidões de perdas que têm experimentado os que tomam as decisões, ante petições externas nas dimensões tecnológicas e ambientais.

Palavras chave: Teoria prospectiva acumulativa; Julgamento perito; Toma de decisões; Megaprojetos; Hidroelétricas.
\end{abstract}


García, R. \& Zerda, A. (2018) Caracterización de la función de valor empleada en las decisiones ambientales por las grandes organizaciones: estudio de los grandes proyectos hidroeléctricos en Colombia. En: Revista de la Facultad de Ciencias Económica: Investigación y Reflexión. rev.fac.cienc.econ, XXVI (1), DOI: https://doi.org/10.18359/rfce.1884

JEL: A12, D80, D81, L20.

\section{Introducción}

La caracterización de la función de valor empleada en las decisiones ambientales por las grandes organizaciones, es una forma de simplificar las decisiones ambientales en función de sus rasgos más relevantes. En este estudio se presenta una aplicación directa en el estudio de las grandes organizaciones responsables de construir las hidroeléctricas más grandes del país con capacidad que supera los 400 MW. En estas organizaciones se realiza una exploración mixta con elementos cualitativos y cuantitativos con el fin de construir un modelo plausible y significativo de la realidad de los procesos de toma de decisiones organizacionales en el campo ambiental.

El modelo se construye tomando como referencia los avances de la teoría prospectiva acumulativa debido a su pertinencia para el campo de decisiones donde los expertos presentan una gran relevancia en las decisiones organizacionales.

La información de campo se recopilo en todas las compañías que promueven mega proyectos hidroeléctricos en Colombia, lo que corresponde en total a 4 empresas: EMGESA, CELSIA, EPM e ISAGEN. Estás compañías son las encargadas de más del 90\% del mercado energético en el país, además se cuentan entre las empresas más grandes presentes en el territorio nacional (Unidad de Planeación Minero Energética-UPME, 2014). Los expertos por su parte, cumplen con los requisitos para ser considerados como tal (Mieg, 2009), como son: excelencia (Alta preparación académica) y profesionalismo (reconocimiento dentro de la organización como experto). La información se presenta en forma agregada. Se establecen dos hipótesis plausibles y generales: homogeneidad de conocimientos y homogeneidad de la organización (Mieg, 2014, pág. 88).
El estudio presenta así los parámetros ajustados del modelo de teoría prospectiva acumulativa y explora las consecuencias en las decisiones organizacionales.

Los resultados son claros y reveladores sobre los sesgos y preferencias de las grandes organizaciones tomadas en cuenta al momento de realizar elecciones en la dimensión ambiental. A partir de los resultados obtenidos se puede inferir las políticas públicas requeridas en este campo con el fin de mejorar su desempeño organizacional.

\section{Antecedentes y literatura existente}

La literatura de interés se centra en aquellas teorías con capacidad de recoger las características encontradas en campo asociadas a las decisiones ambientales dentro de las grandes organizaciones. La primera característica es la presencia de condiciones de incertidumbre, dado la naturaleza de largo plazo de los impactos ambientales, y ambigüedad típica de la construcción de hidroeléctricas, no es posible utilizar modelos donde existan patrones regulares o previsibles. La segunda característica es la simplificación de los modelos de decisión organizacional a través de la delegación en expertos los asuntos ambientales principalmente en los campos de gran ambigüedad y ausencia de información previa. La tercera característica es la amplia adopción de las grandes organizaciones de las normativas en los campos específicos profesionales, lo que profundiza la gran dependencia de los expertos en las interpretaciones de las realidades ambientales de las grandes hidroeléctricas.

Desde el punto de vista teórico se requiere conocer las características de la teoría de toma de decisiones según la aproximación elegida para este estudio. La taxonomía existente de teorías de decisión basadas en juicio experto es presentada en la imagen 1 . 
Imagen 1. Marco de referencia de la teoría de toma de decisiones aplicable al estudio de mega proyectos en el campo de la ingeniería.

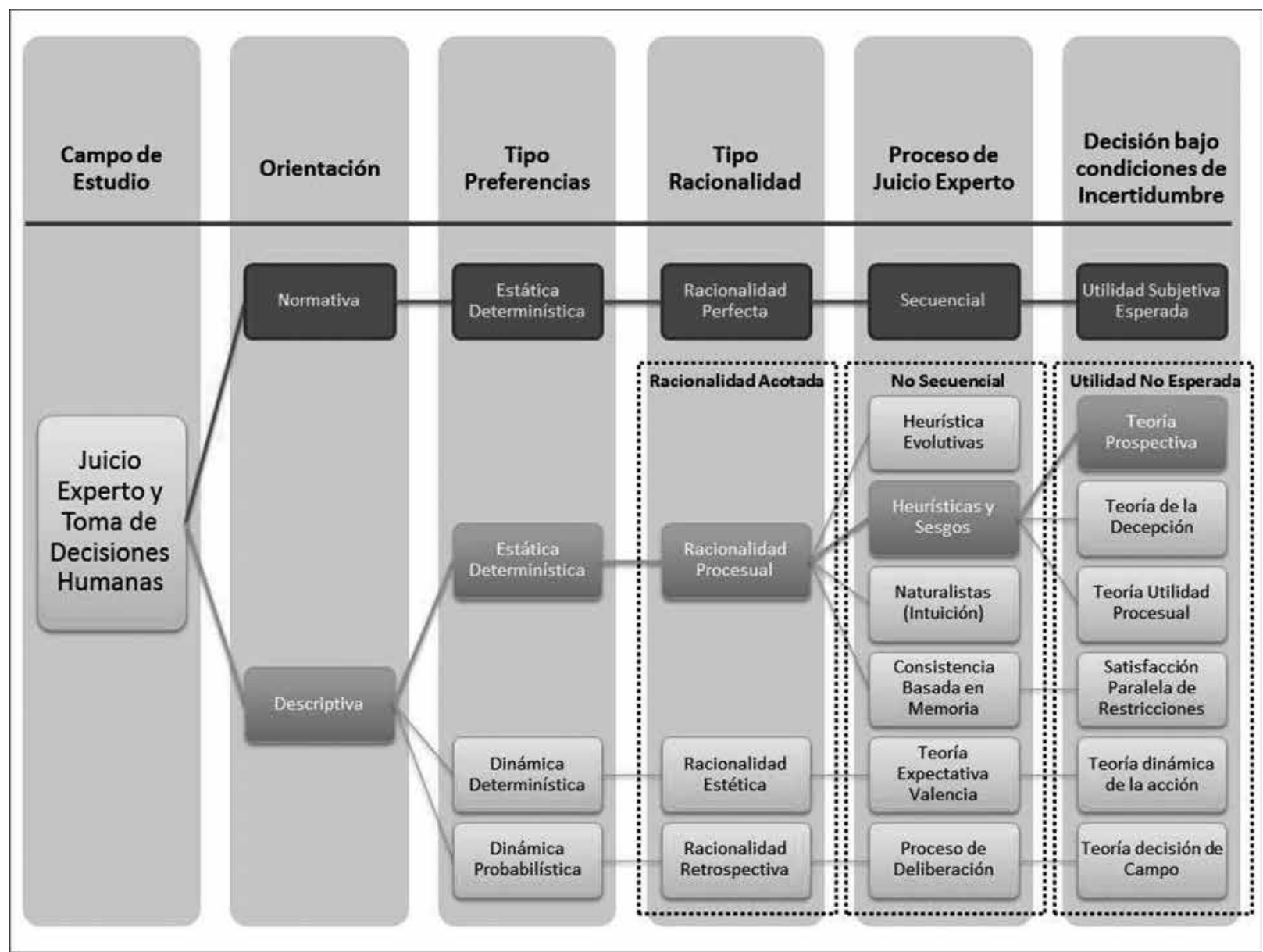

Fuente: elaboración de los autores

Las teorías de toma de decisiones bajo condiciones de incertidumbre aplicables a procesos de grandes organizaciones puede resumirse en siete: Teoría de la Utilidad Esperada (Neumann \& Morgenstern, 1944) (Becker, 1976); Teoría Prospectiva (Tversky \& Kahneman, 1992) ${ }^{1}$; Teoría de arrepentimiento (Loomes \& Sugden, 1982),; Teoría Dual (Yaari, 1987); teoría de satisfacción de restricciones paralelas, Teoría de la Acción Dinámica (Kreps \& Porteus,

$1 \quad$ El término es usado en su concepción moderna con la que se refiere a la Teoría Prospectiva Acumulativa, como se sugiere en el trabajo de Köbberling, V. \& Wakker, P (2003).
1979) y Teoría del Campo de Decisión (Busemeyer \& Townsend, 1993). Por supuesto existen extensiones a estas teorías o cambios en la construcción teórica que no modifican las características particulares dentro de la que se plantea la taxonomía.

Para el estudio presentado se prefiere entre las distintas escuelas que abordan la teoría de toma de decisiones, la Teoría Prospectiva (Tversky \& Kahneman, 1992) dado que coincide con las condiciones del análisis sobre mega proyectos hidroeléctricos en Colombia (Unidad de Planeación Minero Energética-UPME, 2014). Como se ha mencionado 
previamente el trabajo se realiza bajo los presupuestos de la Teoría Prospectiva Acumulativa (Tversky \& Kahneman, 1992), teniendo en cuenta que dicha escuela tiene en cuenta los sesgos y heurísticas (Moritz \& Gieri, 2015) como punto de partida para modelar el proceso de decisión interno de los expertos (Busenitz \& Barney, 1997), principalmente cuando afrontan condiciones de incertidumbre; en adición resulta aplicable a la dimensión de impacto ambiental requerida para estudiar mega proyectos hidroeléctricos (Sovacool \& Cooper, 2013).

\section{Teoría Prospectiva}

Antes de 1979, según lo señala P. Wakker (2010), el comportamiento irracional era visto como caótico e inadecuado para ser modelado. Kahneman \& Tversky (1979) con su Teoría Prospectiva anuncian un panorama más alentador al respecto.

En ésta teoría de elección, a diferencia de la Teoría de la Utilidad Esperada, los valores de ganancia y pérdida se asignan a partir de un punto de referencia, y en cambio de los valores y las probabilidades finales se utilizan rangos de decisión. El valor de la función a diferencia de la Teoría de Utilidad Esperada es cóncavo para las ganancias, convexo para las pérdidas, y como regla general más pronunciado en la zona de pérdida que en la de ganancias. El peso de las decisiones es casi siempre más bajo que el correspondiente a las probabilidades, excepto con rango de probabilidades extremadamente bajo.

La teoría de Kahneman y Tversky demuestra que los individuos tienden a preocuparse por resultados de corto tiempo y su subjetiva función de valor expresa que hay un valor emocional asociado con cambios de riqueza (ganancias o pérdidas) (Kahneman \& Tversky, 1979). La utilidad no puede estar separada de las emociones y éstas a su vez están marcadas por cambios en el contexto de las decisiones (Angner \& Loewenstein, 2007).

La Teoría Prospectiva establece tres parámetros iniciales (Wakker, 2010):

1. Ponderación alta de probabilidades bajas.
2. Ponderación baja de probabilidades medias y altas.

3. Pesos equivalente para ganancias y pérdidas.

Durante la segunda mitad de los 80 `s se observaron comportamientos de riesgo más complejos. Los cuales no podían ser explicados con la Teoría Prospectiva, en consecuencia se asignaron ponderaciones equivalentes para las ganancias y las pérdidas (Wakker, 2010). En búsqueda de superar esta brecha Tversky \& Kahneman (1992) formularon la Teoría Prospectiva Acumulativa.

Los autores argumentaron que la tercera característica que infundió la misma reacción a la pérdida o ganancia por los sujetos de estudio no correspondía con los hallazgos (Wakker, 2010); consecuentemente implementaron una modificación en la que el tercer factor refleja la subjetividad del tomador de decisiones (Tversky \& Kahneman, 1992). Así mismo incluyeron un cuarto parámetro que explicaría el patrón cuádruple de las actitudes riesgosas (Wakker, 2010).

\section{Metodología}

Con el objetivo de desarrollar un modelo descriptivo apropiado sobre toma de decisiones (Holmes Jr., Bromiley, Devers, Holcomb, \& McGuire, 2011) se requirió de un trabajo de campo de observación y captura directa de información en el sitio de trabajo de los expertos (Angner \& Loewenstein, 2007; Charness, Gneezy, \& Imas, 2013). Así, para identificar el proceso de toma de decisiones sobre impactos ambientales ejecutado dentro de los mega proyectos hidroeléctricos ${ }^{2}$, se eligió la entrevista (Ericsson, 2006) semi estructurada como herramienta metodológica, la cual permite abordar variables cuantitativas y cualitativas (Kumar, 2005). El estudio contempla proyectos que exceden la capacidad de generación de 350 MVA (UPME, 2014) desarrollados en Colombia durante el periodo 2010 - 2020. Para diseñar la herramienta metodológica seleccio-

2 Basado en el plan de generación indicativo establecido por UPME 2015-2020 (2014). 
nada fue necesario definir puntos precisos de indagación que resultasen idóneos para corroborar $\mathrm{o}$ negar las afirmaciones presentadas como hipótesis (Ericsson, 2006). De igual forma, la metodología debía permitir la réplica de la información (Charness, Gneezy, \& Imas, 2013) suministrada por los entrevistados además de generar retroalimentación cualitativa que sirviera de validación interna de los datos cuantitativos obtenidos (Hoffman, 1992).

En primera instancia se eligieron las organizaciones sobre las que se realizaría la investigación, las cuales serían las mismas a entrevistar, así tras la revisión de la capacidad de generación y la oferta del servicio (UPME, 2014), se concluyó que las organizaciones: EPM, ISAGEN, EMGESA y CELSIA serían las encargadas de la construcción de todos los mega proyectos hidroeléctricos que se adelantarían en Colombia para el periodo 2010 - 2020. Estas organizaciones delegaron a los expertos (Mieg, 2009) en estudios ambientales correspondientes la responsabilidad de contestar la entrevista propuesta por los autores de este artículo.

Las preguntas fueron exactamente iguales para los cuatro (4) expertos (Hoffman, 1992), se indagaron en el mismo orden y con la misma modalidad, la cual consistía en dar un tiempo de respuesta de máximo 5 minutos al encuestado para su respuesta (Ericsson, 2006). El registro de las respuestas se realizó a través de grabaciones de audio (cuando fueron aprobadas) y toma de notas sobre la primera oración o explicación que enunciaba el experto sobre un tema. Dicho énfasis en la primera reacción del experto garantizó que se extrajera de las encuestas las preocupaciones más inmediatas de las organizaciones en casos específicos (Mieg, 2014). Adicionalmente, la teoría de la toma de decisiones indica que una heurística propia de los expertos consiste en el uso de la disponibilidad en su memoria (Plous, 1993), es decir, que el concepto con que se está más familiarizado lo considera como un ítem más probable dentro de una categoría.

Cada encuesta tuvo una duración aproximada de 30 minutos, de los cuales en un $91 \%$ hablaron los expertos. Las respuestas tenían una única opción en la mayoría de ítems, sin embargo, para conocer las prioridades de las organizaciones (Mieg, 2014), dos de las preguntas solicitaban ordenar de mayor a menor importancia (Plous, 1993) las variables previamente seleccionadas con base en estudios previos sobre evaluación de impacto ambiental (McCormick, 2006, Conesa, 1997; ESI, 2005), y los trabajos previos realizados por otras investigaciones en áreas de estudio cercanas al eje específico de mega proyectos hidroeléctricos (Stone, 2011; Sovacool \& Cooper, 2013; Ansar, Flyvbjerg, Budzier, \& Lunn, 2014).

El primero de los supuesto que indagó la encuesta, fue el de la coincidencia entre la persona que seleccionó cada organización para responder la encuesta y el perfil de experto (Ericsson, 2006). Para verificar este punto se hicieron preguntas en torno a la descripción de la excelencia académica de cada uno y, el reconocimiento del experto dentro de la organización, para esta segunda característica (Hoffman, 1992) se acudió incluso a las referencias de otros agentes intra organización quienes indicaron al mismo sujeto seleccionado para la encuesta como el experto ambiental en mega proyectos hidroeléctricos.

En una segunda etapa se realizaron preguntas que perseguían la confirmación del postulado "No hay desviación entre lo que propone el experto y lo que se ejecuta en cada organización", el cual es uno de los supuestos fundamentales del trabajo de campo (Mieg, 2014), pues evidencia que la organización está plenamente articulada con sus delegados (Moritz \& Gieri, 2015) para la toma de decisiones y que por tanto, el decir de los expertos (Brighton \& Gigerenzer, 2015) refleja la posición e iniciativa organizacional (Holmes Jr., Bromiley, Devers, Holcomb, \& McGuire, 2011) en la dimensión ambiental en los mega proyectos hidroeléctricos.

Para ésta etapa se indagó sobre la aprobación del método de selección y designación de roles en la organización (Holmes Jr., Bromiley, Devers, Holcomb, \& McGuire, 2011), los 4 expertos coincidieron en responder que si estaban de acuerdo con los procesos de asignación, del mismo modo en la ampliación de la respuesta comentaron que se requiere de líderes con conocimientos específicos sobre los 
procedimientos institucionales - ambientales que se deben surtir para iniciar un proyecto hidroeléctrico de gran impacto. Así mismo, los expertos dijeron conocer y estar de acuerdo con los factores que se tiene en cuenta en la elección del equipo, para ello categorizaron los factores dados en la encuesta $(\mathrm{Ku}-$ mar, 2005).

El supuesto de homogeneidad se observó tanto en la información cualitativa como en la cuantitativa, y se refleja en un modo estadístico (DellaVigna, 2009).

La información de carácter cualitativo permite la definición de las variables principales del modelo. Y brinda posteriormente validez interna a las predicciones del modelo cuantitativo en los escenarios de respuesta controlados donde la decisión es conocida y por lo tanto contrastable con la plausibilidad de la caracterización del modelo de decisiones organizacionales revelado.

\subsection{Análisis cualitativo}

Los resultados son presentados primero observando la información de campo y la implicación en el juicio del experto. De este análisis se deriva, el análisis cualitativo de la información dentro del contexto organizacional, considerando la aproximación de organizaciones homogéneas (Mieg, 2014) y orientadas por el criterio experto (Moritz \& Gieri, 2015) utilizando la heurística de la disponibilidad (Plous, 1993) en el ámbito de estudio (Bromiley \& Rau, 2014). Posteriormente, se presenta el análisis cuantitativo realizado, donde se muestran los resultados en la función de valor subjetiva de los expertos y su implicación para la toma de decisiones organizacionales.

Los análisis anteriores permiten comprender el modelo de toma de decisiones ambientales y a partir de estos hallazgos presentar recomendaciones que tienen en cuenta las condiciones de estudio y la contextualización que aporta el análisis cualitativo y cuantitativo realizado.

El esquema de funcionamiento del modelo de toma de decisiones organizacionales, observado en campo y reflejado en la información obtenida permite establecer la existencia de seis (6) características generales en la evaluación de decisiones ambientales:

a). Uso de criterio experto: En las organizaciones, el ámbito de decisión ambiental es responsabilidad del experto ambiental y se observa confianza en su criterio para estos asuntos. Las respuestas mostradas en tabla 1 , reflejan un comportamiento estable y no presentan alta dispersión.

Tabla 1. Respuestas consolidadas asociadas a uso de criterio experto.

\begin{tabular}{|c|c|c|c|c|c|}
\hline PREGUNTAS & $\begin{array}{c}\text { RESPUESTA } \\
\text { TIPO }\end{array}$ & $\begin{array}{c}\text { Experto } \\
\text { Organización A }\end{array}$ & $\begin{array}{c}\text { Experto } \\
\text { Organización B }\end{array}$ & $\begin{array}{c}\text { Experto } \\
\text { Organización C }\end{array}$ & $\begin{array}{c}\text { Experto } \\
\text { Organización D }\end{array}$ \\
\hline $\begin{array}{c}\text { CONSIDERA QUE LA } \\
\text { ORGANIZACIÓN REFLEJA SUS } \\
\text { RECOMENDACIONES TÉCNICAS }\end{array}$ & SI 0 NO & $\mathrm{SI}$ & $\mathrm{SI}$ & $\mathrm{SI}$ \\
\hline RESPONSABILIDAD & TOMA DECISIONES & $\begin{array}{c}\text { Coordinación } \\
\text { Proyectos Plan } \\
\text { de Expansión } \\
\text { Generación } \\
\text { Hidroeléctricas }\end{array}$ & $\begin{array}{c}\text { Coordinación } \\
\text { Ambiental Proyectos } \\
\text { Hidroeléctricos }\end{array}$ & $\begin{array}{c}\text { Jefe Departamento } \\
\text { Medio Ambiente en } \\
\text { EMPRES C S.A. } \\
\text { ESP }\end{array}$ & $\begin{array}{c}\text { Jefe Sistema } \\
\text { Gestión Medio } \\
\text { Ambiental }\end{array}$ \\
\hline $\begin{array}{c}\text { HIDROELÉCTRICAS EN QUE HA } \\
\text { PARTICIPADO }\end{array}$ & $\begin{array}{c}\text { CANTIDAD } \\
\text { PROYECTOS }\end{array}$ & MÁS DE 2 & MÁS DE 2 & MÁS DE 2 & MÁS DE 2 \\
\hline $\begin{array}{c}\text { MÉTODO DE ASIGNACIÓN } \\
\text { EXISTE ES ADECUADO }\end{array}$ & SI O NO & SI & SI & SI & SI \\
\hline
\end{tabular}




\begin{tabular}{|c|c|c|c|c|c|}
\hline PREGUNTAS & $\begin{array}{l}\text { RESPUESTA } \\
\text { TIPO }\end{array}$ & $\begin{array}{c}\text { Experto } \\
\text { Organización A }\end{array}$ & $\begin{array}{c}\text { Experto } \\
\text { Organización B }\end{array}$ & $\begin{array}{c}\text { Experto } \\
\text { Organización } \mathbf{C}\end{array}$ & $\begin{array}{c}\text { Experto } \\
\text { Organización D }\end{array}$ \\
\hline $\begin{array}{c}\text { CONSIDERA QUE EL ESTUDIO } \\
\text { DE IMPACTO AMBIENTAL } \\
\text { DEBE SER REALIZADO POR } \\
\text { EXPERTOS }\end{array}$ & SI o NO & SI & SI & SI & SI \\
\hline $\begin{array}{l}\text { EL RANGO DE PRECISIÓN DE } \\
\text { SUS RECOMENDACIONES ES }\end{array}$ & $\begin{array}{c}D E-50 \% a+100 \% \\
-25 \% a+50 \% \\
-10 \% a+25 \% \\
-5 \% a+10 \%\end{array}$ & Entre $-50 \%$ a $100 \%$ & Entre $-5 \%$ a $10 \%$ & Entre $-50 \%$ a $100 \%$ & Entre $-50 \%$ a $100 \%$ \\
\hline $\begin{array}{l}\text { EL RANGO EN QUE SE ADOPTA } \\
\text { SUS RECOMENDACIONES } \\
\text { TÉCNICAS ES ADECUADO }\end{array}$ & SI o NO & SI & SI & SI & SI \\
\hline
\end{tabular}

Fuente: elaboración de los autores

El resultado es coherente con estudios previos donde el juicio experto presenta alta aceptación en las grandes organizaciones (Earle \& Siegrist, 2008).

b.) Dificultad para percibir la condición de incertidumbre: En las organizaciones se observa el desvanecimiento de las condiciones de incertidumbre; desde su perspectiva interna se afirman en el control y previsión sobre los factores externos sin considerar si han sido comprendidos (Glockner \& Betsch, 2008). Ello implica una delegación de la función de definir estos elementos a los departamentos ambientales. Se observa en la tabla 2 una contradicción entre la capacidad de generar recomendaciones técnicas con alta precisión y la aceptación de la presencia de incertidumbre.

Tabla 2. Respuestas consolidadas asociadas a uso de criterio experto.

\begin{tabular}{|c|c|c|c|c|c|}
\hline PREGUNTAS & $\begin{array}{l}\text { RESPUESTA } \\
\text { TIPO }\end{array}$ & $\begin{array}{c}\text { Experto } \\
\text { Organización A }\end{array}$ & $\begin{array}{c}\text { Experto } \\
\text { Organización B }\end{array}$ & $\begin{array}{c}\text { Experto } \\
\text { Organización C }\end{array}$ & $\begin{array}{c}\text { Experto } \\
\text { Organización D }\end{array}$ \\
\hline $\begin{array}{l}\text { CONSIDERA QUE SE } \\
\text { TOMA DECISIONES } \\
\text { BAJO CONDICIONES DE } \\
\text { INCERTIDUMBRE }\end{array}$ & SI o NO & SI & SI & SI & SI \\
\hline $\begin{array}{c}\text { CONSIDERA ADECUADOS LOS } \\
\text { TIEMPOS QUE ASIGNA LA } \\
\text { ORGANIZACIÓN }\end{array}$ & SI o NO & SI & SI & SI & SI \\
\hline $\begin{array}{l}\text { EL RANGO DE PRECISIÓN DE } \\
\text { SUS RECOMENDACIONES ES }\end{array}$ & $\begin{array}{c}D E-50 \% a+100 \% \\
-25 \% a+50 \% \\
-10 \% a+25 \% \\
-5 \% a+10 \%\end{array}$ & Entre $-50 \%$ a $100 \%$ & Entre $-5 \%$ a $10 \%$ & Entre $-50 \%$ a $100 \%$ & Entre $-50 \%$ a $100 \%$ \\
\hline $\begin{array}{l}\text { EL RANGO EN QUE SE ADOPTA } \\
\text { SUS RECOMENDACIONES } \\
\text { TÉCNICAS ES ADECUADO }\end{array}$ & SI o NO & $\mathrm{SI}$ & SI & SI & SI \\
\hline
\end{tabular}

Fuente: elaboración de los autores 
El resultado es coherente con estudios previos donde la incertidumbre es evitada por las grandes organizaciones (Pettigrew, 1976) y una técnica de mitigación ampliamente aceptada es la adopción del criterio experto (Hofer \& Schendel, 1978). Sin embargo, la decisión ambiental es tomada en un contexto incomprendido (Barandiaran, 2015) debido a la ausencia de los requisitos necesarios para un aprendizaje completo de la realidad (Simon, 1976) en sistemas abiertos como son los sistemas ambientales (Shmelev, 2012). c). Disponibilidad limitada de especialistas: En las organizaciones se identifican grupos compactos dedicados a estudios ambientales; en ningún caso superan los diez (10) profesionales, y solo 2 ó 3 expertos son los referentes en su campo. Los resultados de la tabla 3 indican la presencia de equipos reducidos altamente especializados.

Tabla 3. Respuestas consolidadas de disponibilidad expertos en la organización estudiada.

\begin{tabular}{|c|c|c|c|c|c|}
\hline PREGUNTAS & RESPUESTA & $\begin{array}{c}\text { Experto } \\
\text { TIPO }\end{array}$ & $\begin{array}{c}\text { Experto } \\
\text { Organización A } \\
\text { Organización B }\end{array}$ & $\begin{array}{c}\text { Experto } \\
\text { Organización C }\end{array}$ & $\begin{array}{c}\text { Experto } \\
\text { Organización D }\end{array}$ \\
\hline NOMBRE EXPERTO & $\begin{array}{c}\text { Comentarios } \\
\text { Entrevistados }\end{array}$ & $\begin{array}{c}\text { Se brindan tres } \\
\text { nombres de } \\
\text { expertos internos }\end{array}$ & $\begin{array}{c}\text { Se brinda el nombre } \\
\text { de dos expertos } \\
\text { internos }\end{array}$ & $\begin{array}{c}\text { Se brinda el nombre } \\
\text { de dos expertos } \\
\text { internos }\end{array}$ & $\begin{array}{c}\text { No identifica otros } \\
\text { expertos internos }\end{array}$ \\
\hline $\begin{array}{c}\text { PERSONAL POR PROYECTO } \\
\text { TÍPICO }\end{array}$ & $\begin{array}{c}\text { Observado en sitio } \\
\text { trabajo }\end{array}$ & Menos 10 personas & Menos 10 personas & Menos 10 personas & Menos 10 personas \\
\hline
\end{tabular}

Fuente: elaboración de los autores

Dada la duración de este tipo de proyectos, actualmente estimada entre 7 y 10 años, desde su pre factibilidad hasta su puesta en operación, es un resultado esperado dada la poca disponibilidad de personal calificado dentro de las organizaciones (Boyd \& Banzhaf, 2007). Y, efectivamente, confirma lo sugerido en el estudio de Barandiaran (2015) sobre la dificultad para entes externos de contrastar los resultados de los estudios de impacto ambiental. d). Ausencia de limitaciones internas impuestas por jerarquías o plazos: Los expertos gozan de condiciones adecuadas para opinar; de hecho, en todas las organizaciones se indica total alineación entre las recomendaciones de los expertos ambientales internos y las decisiones corporativas en el campo. Los resultados de la tabla 4, muestran alta similitud entre las respuestas.

Tabla 4. Respuestas consolidadas asociadas a limitaciones a las recomendaciones de los expertos.

\begin{tabular}{|c|c|c|c|c|c|}
\hline PREGUNTAS & $\begin{array}{l}\text { RESPUESTA } \\
\text { TIPO }\end{array}$ & $\begin{array}{c}\text { Experto } \\
\text { Organización A }\end{array}$ & $\begin{array}{c}\text { Experto } \\
\text { Organización B }\end{array}$ & $\begin{array}{c}\text { Experto } \\
\text { Organización } \mathbf{C}\end{array}$ & $\begin{array}{c}\text { Experto } \\
\text { Organización D }\end{array}$ \\
\hline $\begin{array}{l}\text { CONSIDERA ADECUADOS LOS TIEMPOS } \\
\text { QUE ASIGNA LA ORGANIZACIÓN }\end{array}$ & SI o NO & SI & SI & SI & SI \\
\hline $\begin{array}{l}\text { MÉTODO DE ASIGNACION EXISTE ES } \\
\text { ADECUADO }\end{array}$ & SI o NO & NO & SI & SI & SI \\
\hline $\begin{array}{l}\text { CONOCE LOS FACTORES ADOPTADOS } \\
\text { EN LA ORGANIZACIÓN PARA LA } \\
\text { ASIGNACIÓN DE RESPONSABLES DEL } \\
\text { ANÁLISIS DE IMPACTO AMBIENTAL }\end{array}$ & SI o NO & SI & SI & SI & SI \\
\hline TIPO DE PARTICIPACIÓN EN DECISIONES & $\begin{array}{c}\text { TOMA } \\
\text { DECISIONES }\end{array}$ & SI & SI & SI & NO \\
\hline
\end{tabular}

Fuente: elaboración de los autores 
El resultado es contrario a los estudios donde se enfatizan las restricciones de tiempo en la toma de decisiones como condicionante del actuar del experto (Rubenstein, 2013). El resultado apoya el supuesto de organizaciones altamente homogéneas (Mieg, 2014) y ausencia de actuaciones de poder (Goodwin \& Jasper, 1999; Friedl \& Reichl, 2016) como elementos centrales en la comprensión de la toma de decisiones ambientales de los proyectos hidroeléctricos (Myung, 2012).

e). La información primaria es la fuente principal: En estas organizaciones la información primaria es la fuente más confiable de información. Se entiende por información primaria la obtenida en campo directamente por la organización. En este sentido, se desestima la información secundaria externa, y solo es considerada cuando afecta la coherencia de los planteamientos. Los resultados muestran que los expertos prefieren la información directamente recopilada por las organizaciones y desatienden la información secundaria proveniente de otras fuentes. Se presenta una dispersión en las respuestas entre organizaciones.

Tabla 5. Respuestas consolidadas priorización de fuentes de información.

\begin{tabular}{|c|c|c|c|c|c|}
\hline PREGUNTAS & $\begin{array}{c}\text { RESPUESTA } \\
\text { TIPO }\end{array}$ & $\begin{array}{c}\text { Experto } \\
\text { Organización A }\end{array}$ & $\begin{array}{c}\text { Experto } \\
\text { Organización B }\end{array}$ & $\begin{array}{c}\text { Experto } \\
\text { Organización C } \\
\text { Organización D }\end{array}$ \\
\hline $\begin{array}{c}\text { FUENTE INFORMACIÓN } \\
\text { RECOMENDADAS }\end{array}$ & Prioridad 1 & Información primaria & $\begin{array}{c}\text { Comunidades } \\
\text { locales }\end{array}$ & $\begin{array}{c}\text { Información primaria } \\
\text { (estudios propios) }\end{array}$ & $\begin{array}{c}\text { Sistemas de áreas } \\
\text { protegidas }\end{array}$ \\
\hline $\begin{array}{c}\text { FUENTE INFORMACIÓN } \\
\text { RECOMENDADAS }\end{array}$ & Prioridad 5 & Cambio institucional & $\begin{array}{c}\text { Corporaciones } \\
\text { ambientales }\end{array}$ & Medios & $\begin{array}{c}\text { Observatorios } \\
\text { socioeconómicos }\end{array}$ \\
\hline
\end{tabular}

Fuente: elaboración de los autores

El resultado es coherente con el supuesto de la presencia de la heurística de disponibilidad, la cual precisamente privilegia la experiencia propia del experto (Plous, 1993), y asigna mayor validez a la información propia respecto a la obtenida por otras organizaciones externas (Tversky \& Kahneman, 1974). Efectivamente, la tabla 5 muestra que la menor prioridad se asigna a las fuentes secundarias.

f). La estructura organizacional es orientada al proyecto: En las organizaciones estudiadas, los proyectos implican la creación de un grupo independiente con una autonomía alta respecto al funcionamiento de la organización (Barnard, 1968). Los equipos de proyectos son segmentados por fases de maduración de proyectos (March, 1994). Así, los equipos que evalúan los proyectos en etapas de factibilidad no son necesariamente quienes validan los impactos en las etapas de construcción (Flyvbjerg, 2007). 
Tabla 6. Respuestas consolidadas de estructura organizacional.

\begin{tabular}{|c|c|c|c|c|c|}
\hline PREGUNTAS & $\begin{array}{c}\text { RESPUESTA } \\
\text { TIPO }\end{array}$ & $\begin{array}{c}\text { Experto Organización } \\
\mathbf{A}\end{array}$ & $\begin{array}{c}\text { Experto } \\
\text { Organización B }\end{array}$ & $\begin{array}{c}\text { Experto } \\
\text { Organización C }\end{array}$ & $\begin{array}{c}\text { Experto } \\
\text { Organización D }\end{array}$ \\
\hline MEGAPROYECTOS & $\begin{array}{c}\text { Comentarios } \\
\text { EntrevistadoS }\end{array}$ & $\begin{array}{c}\text { Coordinación Proyectos } \\
\text { Plan de Expansión } \\
\text { Generación Hidroeléctricas }\end{array}$ & $\begin{array}{c}\text { Coordinación } \\
\text { Ambiental Proyectos } \\
\text { Hidroeléctricos }\end{array}$ & $\begin{array}{c}\text { Jefe Departamento } \\
\text { Medio Ambiente en } \\
\text { EMPRESA C S.A. ESP }\end{array}$ & $\begin{array}{c}\text { Jefe Sistema } \\
\text { Gestión Medio } \\
\text { Ambiental }\end{array}$ \\
\hline MEGAPROYECTOS & $\begin{array}{c}\text { TOMA } \\
\text { DECISIONES }\end{array}$ & $\mathrm{SI}$ & $\mathrm{SI}$ & $\mathrm{SI}$ \\
$\mathrm{S}$
\end{tabular}

Fuente: elaboración de los autores

El resultado de la tabla 6 , es coherente con el esquema de funcionamiento organizacional previsto para una iniciativa considerada internamente como estratégica (Hofer \& Schendel, 1978), y aislada de la dinámica normal de la operación de la organización (Sovacool \& Cooper, 2013).

Con las características identificadas del proceso, se reconoce un esquema típico aplicado internamente en las organizaciones, por medio del cual se articulan las decisiones del experto con las de la organización. En la imagen 2, se observa el modelo de toma de decisiones aplicado en las organizaciones estudiadas. Los procesos en azul corresponden a procesos ejecutados por los expertos y los procesos en blanco son procesos organizacionales.

El inicio del proceso se da con la recepción de una petición externa a la organización, lo que representa la posibilidad de un cambio para el estudio de impacto ambiental de un proyecto hidroeléctrico. La petición de la comunidad local es asignada dentro de la organización al grupo encargado del proyecto respectivo, y corresponde a este grupo su respuesta formal escrita. La decisión ambiental parte de dos alternativas posibles: a) continuar el estudio de impacto ambiental sin ajustes o b) modificarlo a partir de lo requerido por la comunidad local en su petición externa.

Imagen 2. Modelo descriptivo identificado de toma de decisiones ambientales en las organizaciones

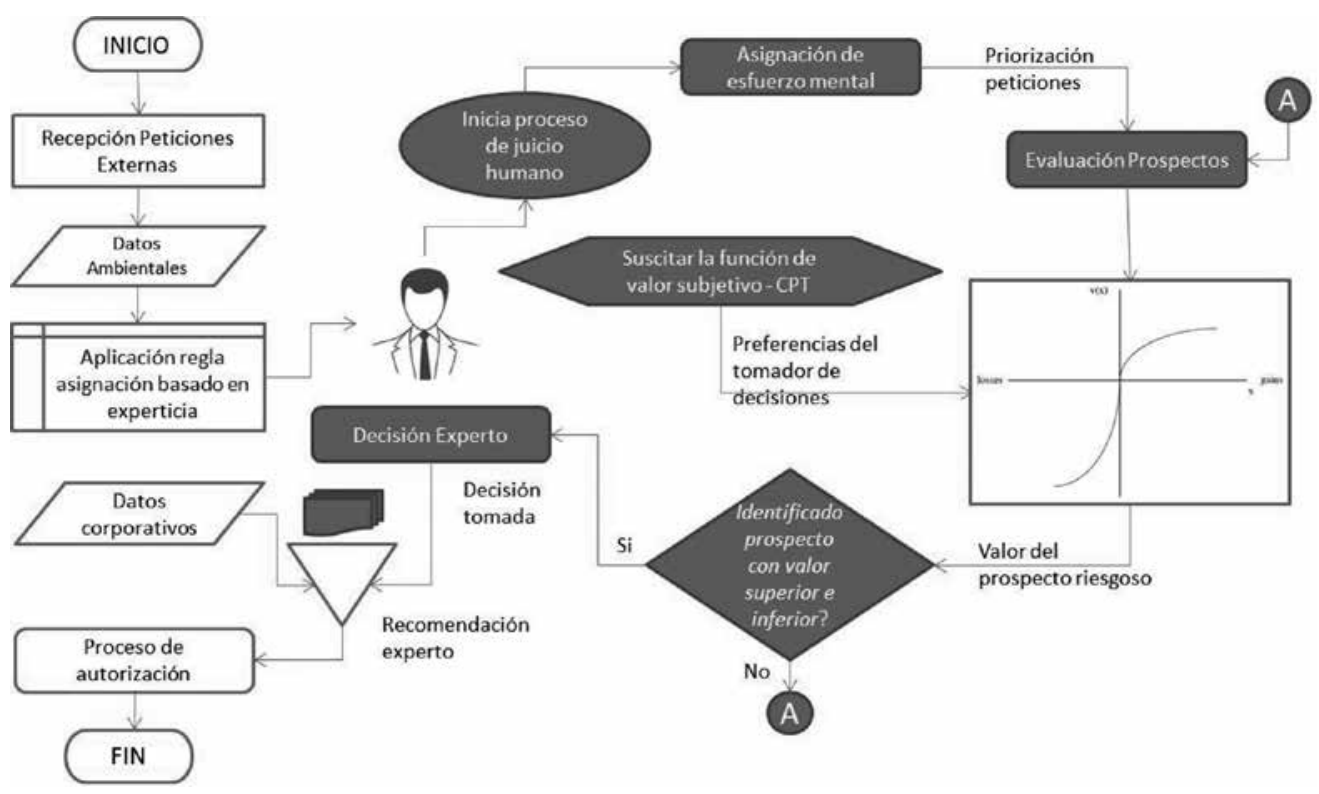

Fuente: elaboración de los autores. 
El modelo de toma de decisiones refleja los procesos basados en juicio de expertos de las organizaciones estudiadas. Los pasos del modelo son los siguientes: 1) se reciben solicitudes externas asociadas a decisiones ambientales de los proyectos hidroeléctricos, 2) dichas peticiones son complementadas con información ambiental relevante, 3) el caso es asignado por la organización a los expertos ambientales basada en criterios de idoneidad, 4) el experto inicia el proceso de juicio experto, 5) primero el experto decide el esfuerzo mental a emplear, 6) valoración de prospectos disponibles en condición de incertidumbre, 7) elección del prospecto según modelo CPT, 8) generación de recomendación del experto a la organización, 9) presentar la información combinada con la información organizacional complementaria, 10) proceso de autorización de la organización previsto para solicitudes externas.

\subsection{Análisis cuantitativo}

Basado en datos recogidos con los cuestionarios (Charness, Gneezy, \& Imas, 2013) la función de valor (actitudes de riesgo) se ha suscitado para cada experto, esto se elaboró teniendo en cuenta el sentido de pérdida o ganancia que tienen las organizaciones hacia las peticiones externas, además a través de la clasificación de acuerdo a las dimensiones que generan situaciones de incertidumbre (Wakker, 2010).

El análisis de la información se ejecutó a través de un primer filtro que consistió en agrupar los resultados según el análisis PESTAL, el cual permite desagregar las condiciones macro ambientales y la situación de una organización (Yüksel, 2012) en torno a los ejes: $(\mathrm{P})$ político, $(\mathrm{E})$ económico, $(\mathrm{S})$ socio cultural, (T) tecnológico, (A) ambiental y (L) legal.

Las categorías identificadas con base en las respuestas dadas por los expertos se muestran en la tabla 7. Alli se consideran las variables de repetición de categorías durante la respuesta de los expertos y la importancia asignada a cada una, del mismo modo se evalúa el tiempo de deliberación usado para responder la pregunta.

Tabla 7. Caracterización de la función de valor en cada organización.

\begin{tabular}{|c|c|c|c|c|c|c|}
\hline $\begin{array}{c}\text { Zona } \\
\text { identificada }\end{array}$ & $\begin{array}{c}\text { Zona del } \\
\text { experto }\end{array}$ & $\begin{array}{c}\text { Organización } \\
\text { de experto A }\end{array}$ & $\begin{array}{c}\text { Organización } \\
\text { de experto B }\end{array}$ & $\begin{array}{c}\text { Organización } \\
\text { de experto C }\end{array}$ & $\begin{array}{c}\text { Organización } \\
\text { de experto D }\end{array}$ & $\begin{array}{c}\text { Ponderación todas } \\
\text { las organizaciones }\end{array}$ \\
\hline \multirow{2}{*}{ Ganancia } & Ganancia Alta & Dimensión social & Dimensión social & $\begin{array}{c}\text { Dimensión } \\
\text { Institucional }\end{array}$ & $\begin{array}{c}\text { Dimensión } \\
\text { Institucional }\end{array}$ & Dimensión social \\
\cline { 2 - 7 } & Ganancia Baja & $\begin{array}{c}\text { Dimensión } \\
\text { Ambiental }\end{array}$ & $\begin{array}{c}\text { Dimensión } \\
\text { Económica }\end{array}$ & $\begin{array}{c}\text { Dimensión } \\
\text { Económica }\end{array}$ & $\begin{array}{c}\text { Dimensión } \\
\text { Tecnológica }\end{array}$ & Dimensión Institucional \\
\hline $\begin{array}{c}\text { Punto de } \\
\text { referencia }\end{array}$ & NEUTRAL & $\begin{array}{c}\text { Dimensión } \\
\text { Económica }\end{array}$ & $\begin{array}{c}\text { Dimensión } \\
\text { Institucional }\end{array}$ & Dimensión social & $\begin{array}{c}\text { Dimensión } \\
\text { Ambiental }\end{array}$ & Dimensión Económica \\
\hline \multirow{2}{*}{\begin{tabular}{c} 
Pérdida \\
\cline { 2 - 7 }
\end{tabular}} & Pérdida Baja & $\begin{array}{c}\text { Dimensión } \\
\text { Institucional }\end{array}$ & $\begin{array}{c}\text { Dimensión } \\
\text { Ambiental }\end{array}$ & $\begin{array}{c}\text { Dimensión } \\
\text { Ambiental }\end{array}$ & Dimensión social & Dimensión Ambiental \\
\cline { 2 - 5 } & Plta & $\begin{array}{c}\text { Dimensión } \\
\text { Tecnológica }\end{array}$ & $\begin{array}{c}\text { Dimensión } \\
\text { Tecnológica }\end{array}$ & $\begin{array}{c}\text { Dimensión } \\
\text { Tecnológica }\end{array}$ & $\begin{array}{c}\text { Dimensión } \\
\text { Económica }\end{array}$ & Dimensión Tecnológica \\
\hline
\end{tabular}

Fuente: elaboración de los autores.

La tabla 7, muestra evidencia sobre como los expertos consideran las dimensiones social $e$ institucional como los factores principales al momento de tomar decisiones de impacto ambiental (Conesa, 1997), por su parte las dimensiones tecnológicas y ambientales no son identificadas por los expertos de forma prioritaria para la toma de decisiones, en este sentido, cuando los expertos reciben peticiones sobre escenarios de incertidumbre que recaen en las dos últimas dimensiones categorizadas, estas son evaluadas en la zona de pérdida de la función de valor (Tversky \& Kahneman, 1992). La zona de ganancia 
se remite a las dimensiones sociales e institucionales. El punto neutro o de referencia se obtiene en la dimensión económica.

Al evaluar la función de valor con las dimensiones anteriormente relacionadas y utilizando los parámetros determinados por los estudios de Bui (2009) para los tomadores de decisiones de Colombia, se evidencia que a pesar de que las dimensiones sociales e institucionales están más presentes en el análisis ambiental de las organizaciones, estos dos escenarios son también negativos en la función de valor que utiliza la teoría prospectiva acumulativa ya que la reacción no es suficiente para sopesar los posibles retos inciertos.

Con el fin de cuantificar el modelo basado en la Teoría Prospectiva Acumulativa, se asignaron pagos ${ }^{3}$ de 100 a la zona de ganancia alta, 50 a la zona de ganancia baja, 0 al punto de referencia, - 50 a la zona de pérdida baja y -100 a la zona de ganancia alta (Charness, Gneezy, \& Imas, 2013).

La fórmula usada sigue el modelo de la Teoría Prospectiva Acumulativa y se demuestra en las siguientes ecuaciones de la 1 a la 5 (Tversky \& Kahneman, 1992).

$$
\begin{gathered}
w^{+}(p)=\frac{p^{\gamma}}{\left(p^{\gamma}+(1-p)^{\gamma}\right)^{\frac{1}{\gamma}}} \\
w^{-}(p)=\frac{p^{\delta}}{\left(p^{\delta}+(1-p)^{\delta}\right)^{\frac{1}{\delta}}} \\
f(x)=\left\{\begin{array}{cc}
x^{\alpha}, \quad \text { if } \alpha>0 \\
\ln x, \quad \text { if } \alpha=0 \\
1-(1+x)^{\alpha}, \quad \text { if } \alpha<0
\end{array}\right.
\end{gathered}
$$

3 Estos valores corresponden a estudios de pago típicos usados en experimentos de economía comportamental (Angner $\&$ Loewenstein, 2007). Además, se realizaron estudios que permitieran analizar escenarios con valores extremadamente bajos y altos. (Holmes Jr., Bromiley, Devers, Holcomb, \& McGuire, 2011).

$$
\begin{gathered}
g(x)=\left\{\begin{array}{cc}
-(-x)^{\beta}, & \text { if } \beta>0 \\
-\ln (-x), & \text { if } \beta=0 \\
(1-x)^{\beta}-1, & \text { if } \beta<0
\end{array}\right. \\
v(x)=\left\{\begin{array}{cc}
f(x), & \text { if } x>0 \\
0, & \text { if } x=0 \\
\lambda \times g(x), & \text { if } x<0
\end{array}\right.
\end{gathered}
$$

El modelo cuantitativo usa la función de poder que recopila las características más importantes entre ellas la dominancia estocástica de segundo orden ${ }^{4}$.

Dado que el modelo apropia información obtenida de expertos colombianos, fue necesario ajustar el modelo a las condiciones culturales del país, por esta razón tres elecciones de parámetros de la Teoría Prospectiva Acumulativa (Tversky \& Kahneman, 1992) son determinantes para el análisis en Colombia según Bui (2009) respeto de la toma de decisiones, los cuales sirvieron de guía para este trabajo.

Los parámetros fueron seleccionados a través del cálculo de la distancia absoluta más cercana desde el modelo original indicado por Tversky y Kahneman.

El modelo llamado "Modelo 111" (Bui, 2009) presenta los coeficientes bajo el criterio seleccionado más idóneos para la utilización en el modelo de caracterización de las organizaciones en Colombia. Los coeficientes obtenidos se muestran en la Tabla 8 y fueron usados para el modelamiento matemático.

Tabla 8. Coeficientes usados en el modelo CPT.

\begin{tabular}{|c|l|c|}
\hline $\begin{array}{c}\text { Símbolos } \\
\text { Parámetro }\end{array}$ & $\begin{array}{c}\text { Valores } \\
\text { asignados }\end{array}$ & Valores originales CPT \\
\hline$\alpha$ & 0,46 & 0.88 (Tversky \& Kahneman, 1992) \\
\hline$\beta$ & 0,88 & 0.88 (Tversky \& Kahneman, 1992) \\
\hline$\lambda$ & 1,74 & 2.25 (Tversky \& Kahneman, 1992) \\
\hline$\gamma$ & 0,69 & 0.61 (Tversky \& Kahneman, 1992) \\
\hline$\delta$ & 0,36 & 0.69 (Tversky \& Kahneman, 1992) \\
\hline
\end{tabular}

Fuente: elaboración de los autores.

4 Una crítica al modelo utilizado puede ser leída en "Birnbaum's paradoxes" (2008). 
El siguiente paso consistió en identificar el vector de probabilidad para Colombia, esto implicó el uso de un modelo preestablecido adecuado para evaluar la condición del país a partir de estas cinco dimensiones, y cuantitativamente representar la función de probabilidad, la cual refleja la presencia de las solicitudes en cada dimensión.

A partir de tres análisis previos, CEPAL (NU. CEPAL, 2015), YCELP (Hsu et al., 2014) y YALE (ESI, 2005) se exploró cuál de ellos contenía información completa con el fin de ponderar todas las dimensiones requeridas en este análisis. Se identificó el modelo de YALE (ESI, 2005) como el más eficaz para soportar la información requerida de Colombia. El modelo seleccionado no requiere hacer conversión de información, lo que asegura que los criterios de los autores no se involucran en la transformación de la información básica.

El vector de probabilidad normalizado para Colombia (ESI, 2005) se encuentra a $19 \%$ para la dimensión social, $24 \%$ para la dimensión ambiental, $18 \%$ para la dimensión económica, $21 \%$ para la dimen- sión institucional y $18 \%$ para la dimensión tecnológica. Estas probabilidades están en el rango medio, lo que indica que el valor de la función no opera en probabilidades extremas (Wakker, 2010).

Con los valores de matriz de pago, coeficientes CPT y vectores de probabilidad se crea una configuración típica de la teoría prospectiva acumulativa de toma de decisiones. En la simulación se incluye un análisis de sensibilidad que se desarrolla usando vectores de probabilidad de $40 \%$ para la dimensión seleccionada como principal y $15 \%$ para el resto de ellas, adicionalmente, se utiliza un vector igual al 20\% para cada dimensión con el fin de validar en rangos diversos los resultados obtenidos de la caracterización del modelo.

\section{Resultados y discusión}

Los resultados obtenidos al aplicar el modelo de la Teoría Prospectiva Acumulativa (Charness, Gneezy, \& Imas, 2013) para los casos de las organizaciones estudiadas en Colombia se muestran en la tabla 9.

Tabla 9. Resultados de la función de valor usando modelo CPT

\begin{tabular}{|c|c|c|c|c|c|c|c|}
\hline \multirow{2}{*}{$\begin{array}{c}\text { Modelo } \\
\text { CPT usando } \\
\text { coeficientes para } \\
\text { Colombia }\end{array}$} & \multicolumn{7}{|c|}{ Función de valor evaluada por caso } \\
\hline & \multicolumn{7}{|c|}{ Análisis de escenarios - Resultados de modelo CPT } \\
\hline $\begin{array}{c}\text { Resultados por } \\
\text { Organización }\end{array}$ & $\begin{array}{c}\text { Caso más } \\
\text { probable para } \\
\text { Colombia }\end{array}$ & $\begin{array}{l}\text { Casos de } \\
\text { relevancia } \\
\text { social }\end{array}$ & $\begin{array}{l}\text { Casos de } \\
\text { relevancia } \\
\text { ambiental }\end{array}$ & $\begin{array}{l}\text { Casos de } \\
\text { relevancia } \\
\text { económica }\end{array}$ & $\begin{array}{l}\text { Casos de } \\
\text { relevancia } \\
\text { institucional }\end{array}$ & $\begin{array}{l}\text { Casos de } \\
\text { relevancia } \\
\text { Tecnológica }\end{array}$ & $\begin{array}{l}\text { Igualdad de } \\
\text { dimensiones }\end{array}$ \\
\hline Organización A & $-16,95$ & $-15,34$ & $-15,74$ & $-16,70$ & $-18,68$ & $-20,15$ & $-17,23$ \\
\hline Organización B & $-17,35$ & $-15,34$ & $-18,68$ & $-15,74$ & $-16,70$ & $-20,15$ & $-17,23$ \\
\hline Organización C & $-17,25$ & $-16,70$ & $-18,68$ & $-15,74$ & $-15,34$ & $-20,15$ & $-17,23$ \\
\hline Organización D & $-16,99$ & $-18,68$ & $-16,70$ & $-20,15$ & $-15,34$ & $-15,74$ & $-17,23$ \\
\hline Consolidado & $-17,26$ & $-15,34$ & $-18,68$ & $-16,70$ & $-15,74$ & $-20,15$ & $-17,23$ \\
\hline
\end{tabular}

Fuente: elaboración de los autores

Lo anterior indica que los tomadores de decisión en grandes organizaciones que adelantan proyectos hidroeléctricos, tienen como característica común la aptitud del riesgo de pérdida frente a los requerimientos enfocados en la dimensión tecnológica (Wakker, 2010). 
También indica una predisposición homogénea de las organizaciones en Colombia a las solicitudes más recurrentes de las comunidades locales (ESI, 2005). En cualquiera de los escenarios analizados los resultados de la función de valor fueron negativos, lo que implica un comportamiento de búsqueda de riesgo en las solicitudes que emergen desde afuera del perímetro organizacional (Bromiley \& Rau, 2014). Los expertos y las organizaciones que adelantan mega proyectos hidroeléctricos en Colombia para el periodo 2010 - 2020, están operando en la zona de pérdida en cualquiera de los escenarios planteados (Moritz \& Gieri, 2015) ${ }^{5}$.
La imagen 3, resume el proceso de toma de decisiones ambientales que realizan las organizaciones a cargo de la implementación de mega proyectos hidroeléctricos en Colombia. El modelo indica fases de deliberación, presentación de recomendaciones y aceptación de la recomendación por parte de la organización, a través de la adopción de la decisión sugerida. El modelo fue diseñado a partir de los hallazgos (DellaVigna, 2009) de la encuesta y la observación del contexto de trabajo (Ericsson, 2006) en el que los expertos ejecutan sus actividades, la cual refleja las dinámicas habituales de las organizaciones (Bromiley \& Rau, 2014).

Imagen 3. Proceso de toma de decisiones ambientales de las grandes organizaciones identificado en campo.

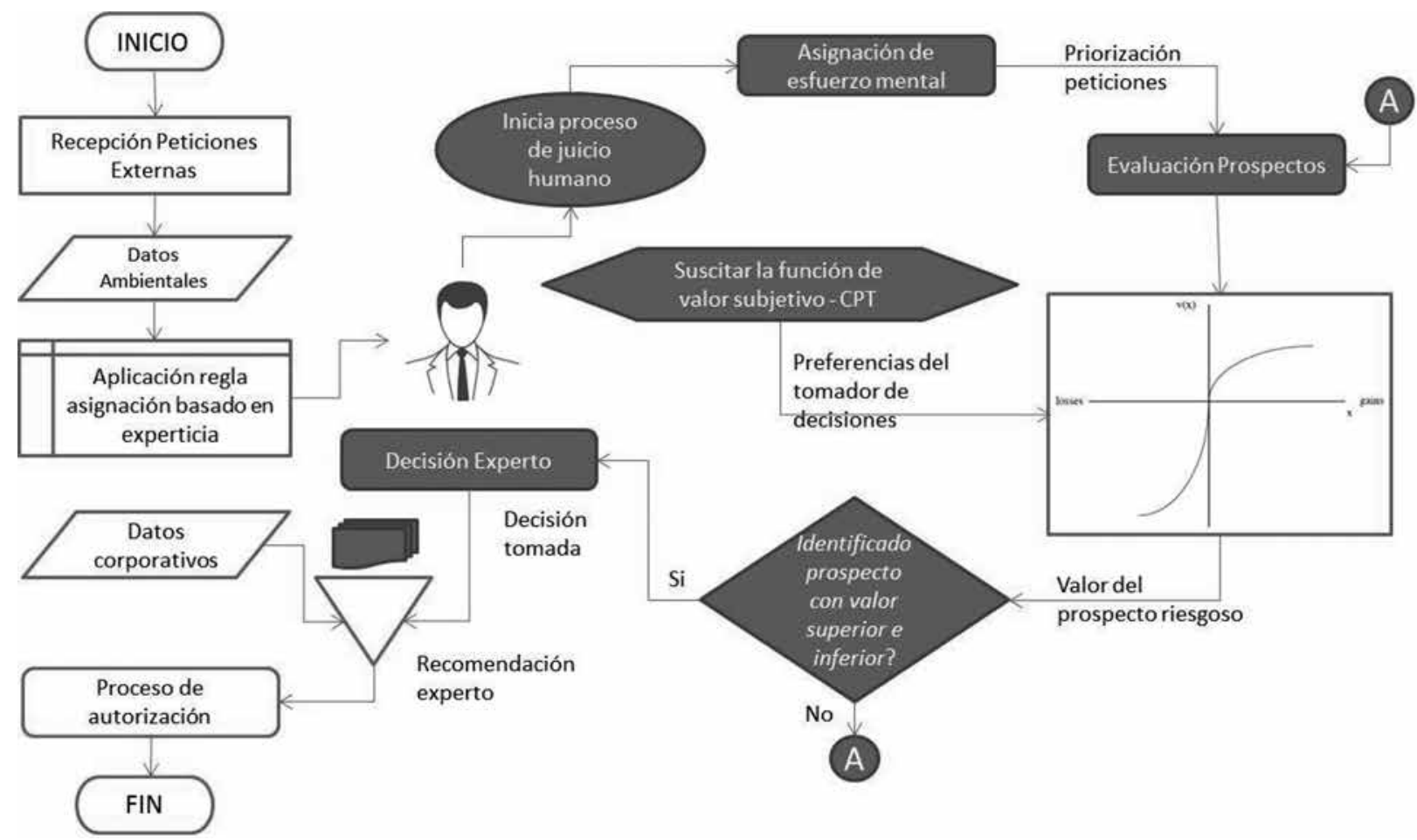

Fuente: elaboración de los autores

5 Vectores de probabilidad que enfatizan en dimensiones sociales, institucionales, económicas, ambientales y tecnológicas. 
El proceso de toma de decisiones inicia con un requerimiento de la comunidad acerca de un mega-proyecto. Con esta solicitud inicial, las organizaciones evalúan la viabilidad de cubrir esta demanda, teniendo en cuenta el grado de impacto ambiental a partir de evaluaciones ya construidas (Conesa, 1997), las reglas de estructura organizacional internas, y el interés económico que tienen en participar en la solución de la demanda insatisfecha (Bromiley \& Rau, 2014). Para determinar la prioridad al proyecto, la organización designa a la unidad de evaluación interna donde se encuentran los expertos ambientales (Holmes Jr., Bromiley, Devers, Holcomb, \& McGuire, 2011).

Una vez que el área correspondiente está a cargo, la persona que lidera el grupo comienza una ponderación interna, en ese momento todos los conocimientos previos del expertos son traídos a colisión, también son utilizados sus acercamientos previos a situaciones similares y la pregunta por las heurísticas presentes es evitada (Brighton \& Gigerenzer, 2015). Los expertos no son conscientes en el momento del comportamiento asumido frente a distorsión de las probabilidades subjetivas (Glockner \& Betsch, 2008).

Del mismo modo, el experto acude a atajos mentales que implican un menor esfuerzo cognitivo para tomar decisiones (Plous, 1993), lo cual corresponde a un proceso ya conocido sustentado por la teoría. El experto toma ventaja de su historia profesional, experticia y conocimientos personales. En este punto es donde la heurística de disponibilidad inmediata hace presencia (Brighton \& Gigerenzer, 2015), esto es, que el experto con base en su conocimiento previo prioriza y organiza la forma en que se conducirá el proceso de toma de decisiones (Moritz \& Gieri, 2015). Cuando el esfuerzo por recordar una dimensión es significativo, el proceso mental del experto asume que es una dimensión que no merece ser considerada como prioritaria, así que se ajusta el punto de referencia para que la experiencia y las heurísticas ubiquen la decisión en un punto de la función de valor que garantice la pérdida (Tversky \& Kahneman, 1992; Bromiley \& Rau, 2014).

El proceso de análisis multidimensional realizado por el experto ocurre de la siguiente manera (Char- ness, Gneezy, \& Imas, 2013): a) Toma cada uno de los escenarios existentes (Shmelev, 2012), b) evalúa los escenarios desde la función de valor, c) a partir de este análisis establece el resultado más probable y la solución alternativa, d) Define la función de riesgo presente en cada escenario e) una vez que el escenario completo es visto en un panorama de ganancia para el experto (que puede ser o no consistente con los datos disponibles externos), se elabora una recomendación final para que la organización la valide y apruebe (Mieg, 2014), f) Para completar el proceso de toma de decisión, la organización compara la recomendación suscrita por el experto con los datos de la compañía para asegurar el cumplimiento de los requisitos mínimos. Finalmente la recomendación se dirige a la organización para ser aprobada o rechazada, en este punto no hay opción de modificar la decisión sin una justificación. (Holmes Jr., et al., 2011).

\subsection{Ajustes al modelo vigente}

A partir de los resultados de la matriz de pagos en toma de decisiones basada en el modelo de la Teoría Prospectiva Acumulativa aplicada con formas nuevas, es posible diseñar recomendaciones para una política mejorada.

El modelo construido en el que se describe el proceso de toma de decisiones constituye una evidencia clara de que el sesgo de disponibilidad inmediata ocurre en el experto delegado cuando aquél está generando la recomendación ambiental de cada organización. Como resultado de este hallazgo, se identifica que cualquier recomendación de cambio debe situarse directamente sobre el esfuerzo mental y la evaluación de las dimensiones ejecutada por el experto.

El modelo también permite observar la afirmación de Kahneman (Gilovich, Griffin, \& Kahneman, 2002) sobre la incapacidad del ser humano de identificar los sesgos de auto confianza y del esfuerzo mental máximo, de tal manera que debe intervenir un agente externo que revise de forma rigurosa los cálculos sobre los valores ${ }^{6}$ ponderados. Con el

6 Conocido como “utilidad” en la Teoría de la Elección Racional (Becker, 1976) 
objetivo de contrarrestar los efectos del sesgo de disponibilidad inmediata en las organizaciones que implementan mega proyectos hidroeléctricos en Colombia, proponemos dos ajustes en la política pública (Bromiley \& Rau, 2014). El primero de ellos consiste en una intervención estatal a través de la política pública para generar una entidad externa a las organizaciones de obligatoria consulta, para construir estudios ambientales que soporten los mega proyectos (Boyd \& Banzhaf, 2007). El segundo consiste en la creación de un cuerpo al interior de las organizaciones (Flyvbjerg, 2007) que valide los resultados indicados en las recomendaciones, que en última instancia servirá como control previo a la aplicación del mega proyecto hi- droeléctrico propuesto (UN, EC, IMF, OECD and World Bank, 2014).

Estos ajustes implican asumir un modelo del proceso de toma de decisiones ambientales, en los cuales: a) la intervención de las autoridades requiere la disponibilidad de datos con antelación usando "cambios de desencadenas" (Bromiley \& Rau, 2014, pág. 21), y b) los nuevos pasos por los que pasará la revisión ambiental aseguren la respectiva validación basándose en "Diseños de Comportamiento Institucional" (Thaler \& Benartz, 2004) (DellaVigna, 2009) y "Creación de Oportunidades para el Cambio" (Bromiley \& Rau, 2014, p.p. 22-23). El modelo se presenta en la imagen 4.

Imagen 4. Propuesta de modelo de toma de decisiones ambientales bajo incertidumbre adoptado por grandes organizaciones.

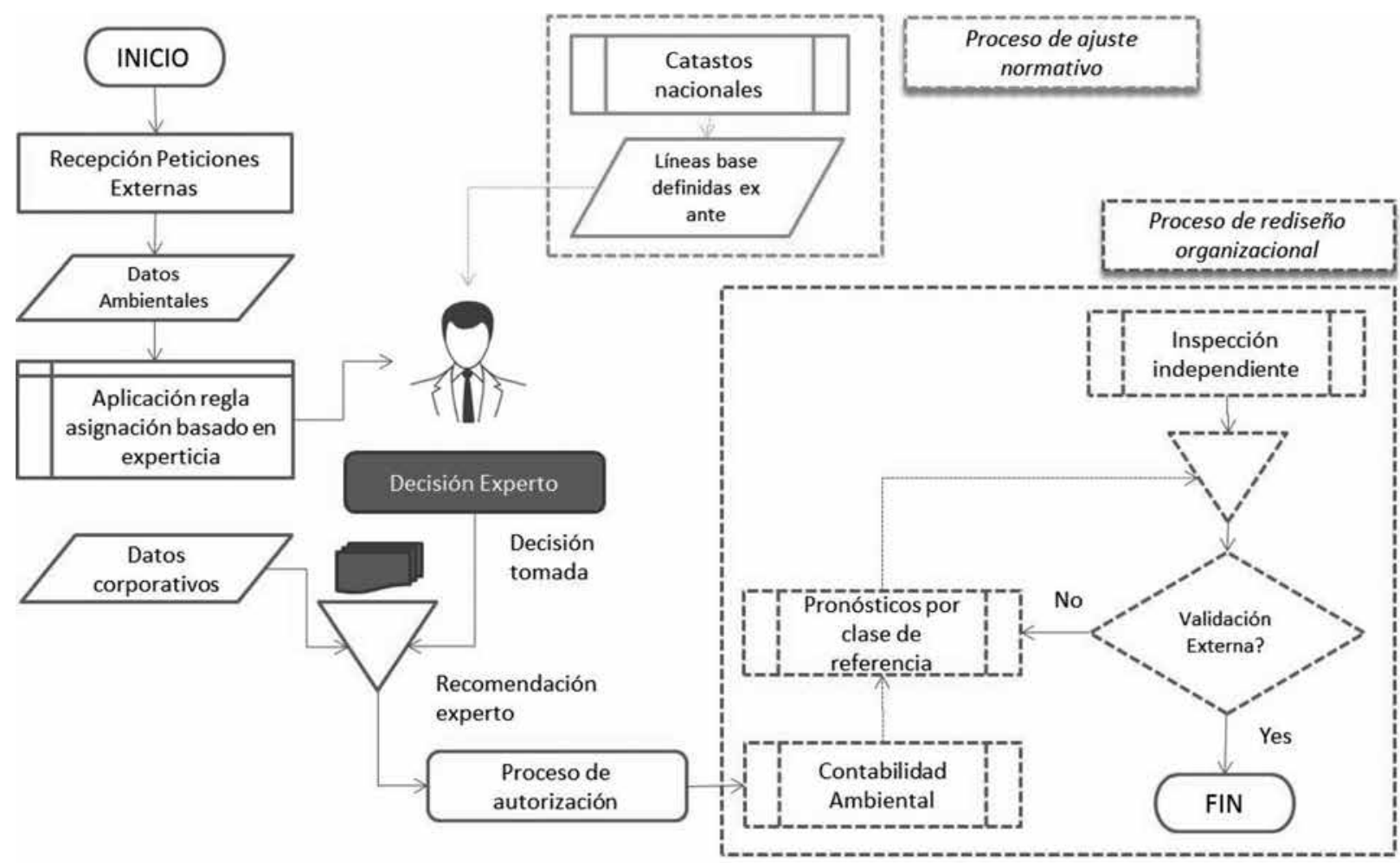

Fuente: elaboración de los autores 
El nuevo modelo presenta cambios en el proceso interno de deliberación seguido por los expertos ${ }^{7}, y$ en el momento que inicia la actividad para generar recomendaciones. El equipo guiado por cada experto debe acudir a datos generados externamente a la organización, que se recopilan por entidades estatales. La comunidad a su vez puede participar desde diferentes áreas de control y verificar si la información final recogida en la recomendación utiliza las líneas de base ambientales obligatorias ${ }^{8}$.
Después de la deliberación del experto basado en los datos obtenidos a través de consolidados externos, la fase de recomendación incorpora estos dos nuevos pasos (Bromiley \& Rau, 2014): 1) una verificación interna de los datos a través de la contabilidad ambiental (Odum, 1996) y 2) previsión de la referencia de clase (Flyvbjerg, 2007), donde las organizaciones pueden asegurar el cumplimiento de la "verificación externa".

Tabla 10. Resultado de la función de valor usando modelo CPT

\begin{tabular}{|c|c|c|c|c|c|c|c|}
\hline \multirow{2}{*}{$\begin{array}{c}\text { Modelo } \\
\text { CPT usando } \\
\text { coeficientes para } \\
\text { Colombia }\end{array}$} & \multicolumn{7}{|c|}{ Valores de función evaluados por caso } \\
\hline & \multicolumn{7}{|c|}{$\begin{array}{l}\text { ESCENARIO DE ANÁLISIS - RESULTADOS DE MODELO CPT - CON RECOMENDACIONES } \\
\text { INCORPORADAS }\end{array}$} \\
\hline $\begin{array}{l}\text { Resultados por } \\
\text { organización }\end{array}$ & $\begin{array}{l}\text { Caso más } \\
\text { probable en } \\
\text { Colombia }\end{array}$ & $\begin{array}{l}\text { Casos de } \\
\text { relevancia } \\
\text { social }\end{array}$ & $\begin{array}{l}\text { Casos de } \\
\text { relevancia } \\
\text { ambiental }\end{array}$ & $\begin{array}{l}\text { Casos de } \\
\text { relevancia } \\
\text { económico }\end{array}$ & $\begin{array}{l}\text { Casos de } \\
\text { relevancia } \\
\text { institucional }\end{array}$ & $\begin{array}{l}\text { Casos de } \\
\text { relevancia } \\
\text { tecnológico }\end{array}$ & $\begin{array}{l}\text { Igualdad de } \\
\text { dimensiones }\end{array}$ \\
\hline Organización A & $-8,20$ & $-6,59$ & $-8,04$ & $-8,04$ & $-9,79$ & $-8,04$ & $-8,09$ \\
\hline Organización B & 2,84 & 3,83 & 2,47 & 3,43 & 2,47 & 2,47 & 2,95 \\
\hline Organización C & 2,94 & 2,47 & 2,47 & 3,43 & 3,83 & 2,47 & 2,95 \\
\hline Organización D & $-17,72$ & $-19,35$ & $-17,37$ & $-20,82$ & $-15,91$ & $-17,37$ & $-18,05$ \\
\hline $\begin{array}{l}\text { Consolidado todas las } \\
\text { organizaciones }\end{array}$ & 2,93 & 3,83 & 2,47 & 2,47 & 3,43 & 2,47 & 2,95 \\
\hline
\end{tabular}

Fuente: elaboración de los autores

Aplicando los ajustes propuestos para el modelo $\mathrm{CPT}$ que usan las organizaciones en la toma de decisiones, se observa un cambio significativo en la función de valor. La tabla 10, presenta los resultados que se obtendrían en la función de valor si se incorporan las recomendaciones.

7 "...Prospect theory would suggest that framing the status quo as a situation that could lead to a loss could trigger more risk taking than presenting the current situation as leading to a gain..." (Bromiley \& \& Rau, 2014, p. 21).

8 Nuestros resultados coinciden con el Banco Mundial: “...It is clear that further advancement in terms of concepts and theory is required; ..., the development and testing of measurement methods is needed..." (UN, EC, IMF, OECD and World Bank, 2014, p.p. 2-4)
Los resultados son sorprendentes, tal como se presenta en la Tabla 11, para la mitad de las organizaciones encuestadas los cambios sugeridos implican cambiar su zona de operaciones, de la zona de altas pérdidas a la de ganancias bajas (Dohmen, Falk, Huffman, \& Sunde, 2011). Una organización permanece en la zona de pérdida aunque con un valor menor. Solo una organización desmejora su situación y aumenta su exposición a la zona de pérdida. Los resultados indican que estás recomendaciones vistas desde un acercamiento general (Heath, Larrick, \& Klayman, 1998) pueden disminuir el conflicto típico entre las comunidades locales y las grandes organizaciones que están a cargo de implementar proyectos hidroeléctricos en Colombia. 
Tabla 11. Resultados de la función de valor usando modelo CPT.

\begin{tabular}{|c|c|c|c|c|c|c|c|}
\hline \multirow{3}{*}{$\begin{array}{c}\text { Modelo } \\
\text { CPT usando } \\
\text { coeficientes para } \\
\text { Colombia }\end{array}$} & \multicolumn{7}{|c|}{ Valores de función evaluados por caso } \\
\hline & \multicolumn{7}{|c|}{ ESCENARIO DE ANÁLISIS - RESULTADOS MODELO CPT - PORCENTAJE DE MEJORA } \\
\hline & $\begin{array}{l}\text { Caso más } \\
\text { probable en } \\
\text { Colombia }\end{array}$ & $\begin{array}{l}\text { Casos de } \\
\text { relevancia } \\
\text { social }\end{array}$ & $\begin{array}{l}\text { Casos de } \\
\text { relevancia } \\
\text { ambiental }\end{array}$ & $\begin{array}{l}\text { Casos de } \\
\text { relevancia } \\
\text { económico }\end{array}$ & $\begin{array}{l}\text { Casos de } \\
\text { relevancia } \\
\text { institucional }\end{array}$ & $\begin{array}{l}\text { Casos de } \\
\text { relevancia } \\
\text { tecnológico }\end{array}$ & $\begin{array}{l}\text { Igualdad de } \\
\text { dimensiones }\end{array}$ \\
\hline Organización A & $52 \%$ & $57 \%$ & $49 \%$ & $52 \%$ & $48 \%$ & $60 \%$ & $53 \%$ \\
\hline Organización B & $116 \%$ & $125 \%$ & $113 \%$ & $122 \%$ & $115 \%$ & $112 \%$ & $117 \%$ \\
\hline Organización C & $117 \%$ & $115 \%$ & $113 \%$ & $122 \%$ & $125 \%$ & $112 \%$ & $117 \%$ \\
\hline Organización D & $-4 \%$ & $-4 \%$ & $-4 \%$ & $-3 \%$ & $-4 \%$ & $-10 \%$ & $-5 \%$ \\
\hline $\begin{array}{l}\text { Consolidado todas las } \\
\text { organizaciones }\end{array}$ & $117 \%$ & $125 \%$ & $113 \%$ & $115 \%$ & $122 \%$ & $112 \%$ & $117 \%$ \\
\hline
\end{tabular}

Fuente: elaboración de los autores

\section{Conclusiones}

El estudio logra proponer una aproximación teórica y metodológica novedosa para comprender y caracterizar los modelos de toma de decisión organizacionales al realizar megaproyectos como son las grandes hidroeléctricas en Colombia en el período analizado.

Las organizaciones que lideran la construcción de hidroeléctricas en Colombia, están categorizadas como grandes organizaciones, de hecho, los proyectos hidroeléctricos son consideradas por estas como elementos de crecimiento orgánico dentro de la etapa de transformación ya que la generación de energía basada en tecnología avanzada, corresponde a la actividad principal de sus nichos de mercado. Como ha sido estudiado, estas organizaciones se caracterizan por ejecutar actividades en las que el proceso de toma de decisiones se basa en las recomendaciones de expertos (Garcia \& Zerda, 2016).

Para desarrollar varios proyectos de forma paralela las grandes organizaciones delegan responsabilidades bajo el criterio de especialidad a los expertos a cargo de sus principales áreas, esto lo hacen en busca de minimizar riesgos y aumentar su capacidad de generar utilidades.
Para la Teoría Prospectiva el mecanismo por el que la carga de toma de decisiones es asignado al personal altamente calificado determina las consecuencias y los costos generados por cada alternativa, lo cual no es un factor superable a nivel individual, sin embargo, debe ser tenido en cuenta como una constante no permanente que puede variar según el contexto y sentimiento de los individuos intervinientes.

Determinar el daño ambiental de la ejecución de un proyecto en un solo lugar, a menudo es uno de los aspectos que los expertos dan por cierto, incluso esos datos son expuestos a las comunidades de una u otra manera para conseguir la aprobación social o cumplir con el requisitos tales como las conocidas las consultas previas. El panorama completo en el que se van a consolidar las afectaciones a la comunidad a partir de la construcción de un mega proyecto hidroeléctrico no puede ser descifrado al momento de la toma de decisiones, entre otras razones porque los efectos ambientales van a ser visibles luego de muchos años, décadas o incluso siglos después de su implementación.

Pero a pesar de la incertidumbre que rodea este tipo de mega proyectos, los expertos (quienes representan a las grandes organizaciones) no perciben la volatilidad de la información en la que basan sus de- 
cisiones. La naturaleza humana al parecer dirigida a controlar todas las variables en un futuro incierto, sigue generando relaciones controversiales y hostiles entre organizaciones, el Estado y la comunidad durante la ejecución de este tipo de proyectos.

Los conflictos que enfrentan los agentes se gestan por una parte en la construcción de los mega-proyectos y por otra al ocultar información en la socialización con las comunidades. Las comunidades locales continúan siendo vistas por los tomadores de decisiones de las grandes organizaciones a cargo de la construcción de mega proyectos de ingeniería, como adversos a este tipo de iniciativas, por lo que las relaciones entre unos y otros resultan normalmente conflictivas. Todo esto ocurre en un ambiente de ambigüedad y profunda complejidad (Ansar, Flyvbjerg, Budzier \& Lunn, 2014) donde la estructuración de grandes proyectos de ingeniería va más allá de las predicciones de los estudios de impacto ambiental, tal como ocurre con la minería hoy en día.

A partir de los resultados de la caracterización (con las novedades insertadas por esta investigación) de este proceso de toma de decisiones basada en el modelo de la Teoría Prospectiva Acumulativa, generan nuevas formas de diseñar recomendaciones sobre la política pública energética totalmente refrescante respecto a las interpretaciones teóricas existentes.

Los resultados evidencian que los expertos son buscadores de riesgos. Las organizaciones que delegan el proceso de toma de decisiones ambientales al criterio de sus expertos cuando se trata de mega proyectos hidroeléctricos, tienen un comportamiento predecible como buscadores de riesgos porque los expertos presentan pérdidas al afrontar solicitudes externas de la comunidad. Este comportamiento se vuelve relevante cuando trata sobre dimensiones ambientales o tecnológicas que necesitan ser ponderadas para dar trámite a requerimientos de las comunidades. Los sesgos descritos de los expertos deben ser corregidos a través de ajustes organizacionales, como los propuestos por los autores de este artículo.

Como ha sido demostrado la razón para los niveles de conflictividad actual se pueden explicar con la aceptación de las causas endógenas de la organización (Bromiley \& Rau, 2014), siendo los modelos de toma de decisiones basadas en expertos una razón primordial (Ansar, Flyvbjerg, Budzier \& Lunn, 2014). Por esta razón se justifica la transformación de la política pública para que limite los conflictos $y$ los recursos destinados a resolver controversias de forma que sean mínimos, en lugar de habituales como en la actualidad.

Para las organizaciones hay dimensiones subestimadas al momento de tomar decisiones ambientales (Holmes Jr., Bromiley, Devers, Holcomb, \& McGuire, 2011), lo que implica procesos de conciliación en estados más avanzados de la implementación de los proyectos con un costo más alto tanto para las organizaciones como para las comunidades (Moritz \& Gieri, 2015). Las generaciones de políticas públicas que aseguren procesos de maduración adecuada de los proyectos, teniendo en cuenta estos nuevos hallazgos en el mercado eléctrico colombiano son necesarias. En una etapa preliminar los autores han explorado un modelo diferente de proyectos en Chile (que se asemeja al propuesto en este estudio) y se han encontrado los mismos resultados, así que es posible que emerjan nuevos campos de investigación para validar estos conceptos en la implementación de mega proyectos.

La evaluación ambiental sostenible (Ansar, Flyvbjerg, Budzier, \& Lunn, 2014) implica la igualdad de trato dentro de las dimensiones con el fin de evitar su contextualización con la realidad de cada proyecto. Si estos elementos no se abordan dentro de las organizaciones, la posibilidad real de influencia de las comunidades entendida como igualdad de trato en las diversas categorías de solicitudes se reduce y por lo tanto también su participación temprana.

Finalmente, la situación encontrada requiere la exploración de políticas energéticas (Sovacool \& Cooper, 2013) fijadas para dirigir la toma de decisiones a propósitos de desarrollo sostenible desde el campo de estudio de las organizaciones (Bromiley \& Rau, 2014) y su operación en el marco de las instituciones en Colombia. Siendo las políticas públicas encaminadas a abordar la necesidad de vincular los catastros ambientales nacionales con las líneas ba- 


\section{CARACTERIZACIÓN DE LA FUNCIÓN DE VALOR EMPLEADA EN LAS DECISIONES AMBIENTALES POR LAS GRANDES ORGANIZACIONES: ESTUDIO DE LOS GRANDES PROYECTOS HIDROELÉCTRICOS EN COLOMBIA}

ses ambientales de los megaproyectos lo más prioritario. A nivel organizacional, urgen cambios en los modelos de toma decisiones donde la organización tenga oportunidad real de enriquecer el juicio experto disponible considerando las debilidades de tipo general descritas en este estudio.

\section{Referencias}

Alos-Ferrer, C., \& Strack, F. (2014). From dual processes to multiple selves: Implications for economic behavior. Journal of Economic Psychology, 41, 1-11.

Angner, E., \& Loewenstein, G. (2007). Behavioral Economics. En M. Uskali, Handbook of the philosophy of science: Philosophy of Economic. 5, p. 641-690. Amsterdam: Elsevier.

Ansar, A., Flyvbjerg, B., Budzier, A., \& Lunn, D. (March de 2014). Should We Build More Large Dams? The Actual Costs of Hydropower Megaproject Development. Energy Policy, 1-14.

Atkinson, J. W., \& Birch, D. (1970). The dynamics of action. New York: Wiley.

Atkinson, R., \& Crawford, L. (2006). Fundamental uncertainties in projects and the scope of project management. International Journal Project Management, 687-698.

Bakka, J. F., \& Lindkvist, L. (1999). Organisationsteori: struktur, kultur, processer (3 ed.). Malmø, Sweden: Liber AB.

Barnard, C. I. (1968). The Functions of the Executive (30 ed.). Cambridge, MA: Harvard University Press.

Becker, G. S. (1976). The Economic Approach to Human Behavior. Chicago: University of Chicago Press.

Birnbaum, M. H. (2008). New paradoxes of risky decision making. Psychological Review, 115(2), 453-501.

Boyd, J., \& Banzhaf, S. (2007). What Are Ecosystem Services? The Need for Standardized Environmental Accounting Units. Ecological Economics, 63, 616-626.

Brighton, H., \& Gigerenzer, G. (2015). The bias bias. Journal of Business Research, 68(8), 1772-1784.

Bromiley, P., \& Rau, D. (2014). How Would Behavioral Strategy Research Lead to Prescription? Journal of Business Economics, 84, 5-25.

Bui, T. (2009). Prospect theory and functional choice. Bielefeld: Bielefeld University, Germany; The University of Paris 1 Pantheon-Sorbonne, France.

Burghart, D., Glimcher, P. W., \& Lazzaro, S. (2013). An Expected Utility Maximizer Walks Into A Bar. Journal of Risk and Uncertainty, 215-246.

Busemeyer, J. R., \& Townsend, J. T. (1993). Decision Field Theory: A dynamic cognition approach to decision making. Psychological Review, 432-459.

Busenitz, L. A., \& Barney, J. B. (1997). Differences between entrepreneurs and managers in large organizations: Biases and heuristics in strategic decision-makin. Journal of Business Venturing, 12, 9-30.
Camerer, C. F., \& Hogarth, R. M. (1999). The effects of financial incentives in experiments: A review and capital-labor-production framework. Journal of Risk and Uncertainty, 19, 7-42.

Censi, M., Corradini, M., Feduzi, A., \& Ghenoa, A. (2015). Halffull or half-empty? A model of decision making under risk. Journal of Mathematical Psychology, 68-69.

Charness, G., Gneezy, U., \& Imas, A. (2013). Experimental methods: Eliciting risk preferences. Journal of Economic Behavior \& Organization, 87, 43- 51.

Conesa, F. V. (1997). Guía metodológica para la evaluación del impacto ambiental. (Tercera ed.). Madrid España: Ediciones Mundi-Prensa.

DellaVigna, S. (2009). Psychology and Economics: Evidence from the Field. Journal of Economic Literature, 315-372.

Dohmen, T., Falk, A., Huffman, D., \& Sunde, U. (2011). Individual risk attitudes: measurement, determinants and behavioral consequences. Journal of the European Economic Association, 522-550.

Earle, T., \& Siegrist, M. (2008). Trust, Confidence and Cooperation model: a framework for understanding the relation between trust and Risk Perception. International Journal of Global Environmental Issues, 8, 17-29.

Ericsson, K. A. (2006). Protocol Analysis and Expert Thought: Concurrent Verbalizations of Thinking during Experts' Performance on Representative Tasks. En N. Charness, P. J. Feltovich, R. R. Hoffman, \& K. A. Ericsson, The Cambridge Handbook of Expertise and Expert Performance (págs. 223-231). Cambridge: Cambridge University Press.

ESI. (2005). Environmental Sustainability Index. Appendix B Country Profiles. New Haven: Yale University's, Center for Environmental Law and Policy.

Flyvbjerg, B. (March de 2007). Policy and Planning for LargeInfrastructure Projects: Problems, Causes, Cures. Environment and Planning B: Planning and Design, 34(4), 578-597.

Flyvbjerg, B., Holm, M. S., \& Soren, L. (2005). How (In)accurate Are Demand Forecasts in Public Works Projects? The Case of Transportation. Journal of the American Planning Association, 131-146.

Garcia, R., \& Zerda, A. (2016). Tesis de Grado No Publicada: Toma de decisiones por grandes organizaciones en condiciones de Incertidumbre: Estudio de las grandes hidroeléctricas en Colombia 2010-2020. Bogota: UN Publicaciones.

Gigerenzer, G. (2015). Simply rational: Decision making in the real world. New York: Oxford University Press.

Gigerenzer, G., Hertwig, R., \& Pachur, T. (2011). Heuristics: The foundations of adaptive behavior. New York: Oxford.

Gilovich, T., Griffin, D. W., \& Kahneman, D. (2002). Heuristics and Biases: The Psychology of Intuitive Judgment. New York: Cambridge University Press.

Glockner, A., \& Betsch, T. (2008). Do people make decisions under risk based on ignorance? Organizational Behavior and Human Decision Processes, 107, 75-95.

Glöckner, A., \& Betsch, T. (2012). Decisions beyond boundaries: When more information is processed faster than less. Acta Psychologica, 139, 532-542. 
Goodwin, J., \& Jasper, J. M. (1999). Caught in a Winding, Snarling Vine: The Structural Bias of Political Process Theory. Sociological Forum, 14, 27-92.

Hansson, S. O. (1994). Decision Theory, a brief introduction. Stockholm: Royal Institute of Technology (KTH).

Hausman, D. M. (2012). Preference, value, choice, and welfare. New York: Cambridge University Press.

Heath, C., Larrick, R. P., \& Klayman, J. (01 de 01 de 1998). CiteSeerx. Recuperado el 17 de 10 de 2015, de Cognitive repairs: How organizational practices can compensate for individual shortcomings (1998): http://citeseerx.ist.psu.edu/ viewdoc/summary?doi=10.1.1.110.5562

Hofer, C. W., \& Schendel, D. (1978). Strategy formulation: Analytical concepts. St. Paul: West Pub. Co.

Hoffman, R. R. (1992). The Psychology of expertise: Cognitive research and empirical AI. New York: Springer-Verlag.

Holmes Jr., R., Bromiley, P., Devers, C., Holcomb, T., \& McGuire, J. (July de 2011). Management theory applications of prospect theory: Accomplishments, challenges, and opportunities. Journal of Management, 37 (4), 1069-1107.

Hsu, A., Emerson, J., Levy, M., Sherbinin, A. d., Johnson, L., Malik, O., y otros. (2014). The 2014 Environmental Performance Index. New Haven, CT: Yale Center for Environmental Law and Policy.

Jawahar, I. M., \& McLaughlin, G. L. (2001). Toward a Descriptive Stakeholder Theory: An Organizational Life Cycle Approach. The Academy of Management Review, 26, 397-414.

Kahneman, D., \& Klein, G. (2009). Conditions for intuitive expertise: A failure to disagree. American Psychologist, 64, 515-526.

Kahneman, D., \& Tversky, A. (1979). Prospect theory: An analysis of decisions under risk. Econometrica, 47(2), 313-327.

Kirkebøen, G. (2009). Decision behaviour - Improving expert judgement. En W. T.W., S. K., \& S. K.J., Making essential choices with scant information : front-end decision making in major projects. (págs. 169-194). New York: PalgraveMacmillan.

Klaes, M., \& Sent, E.-M. (2005). A Conceptual History of the Emergence of Bounded Rationality. History of Political Economic, 37, 27-59.

Knight, F. H. (1921). Risk, Uncertainty, and Profit. New York: Houghton Mifflin.

Köbberling, V., \& Wakker, P. P. (2003). Preference Foundations for Nonexpected Utility: A Generalized and Simplified Technique. Mathematics of Operations Research, 28, 395-423.

Kreps, D. M., \& Porteus, E. L. (1979). Dynamic Choice Theory and Dynamic Programming. Econometrica, 47, 91-100.

Kuhn, T. (1962). The Structure of Scientific Revolutions (First ed.). Chicago: The University of Chicago Press.

Kumar, R. (2005). Research Methodology-A Step-by-Step Guide for Beginners (Second ed.). Singapore: Pearson.

Loomes, G., \& Sugden, R. (1982). Regret theory: An alternative theory of rational choice under uncertainty. Economic Journal, 92, 805-824.
Lovallo, D., \& Kahneman, D. (July de 2003). Delusions of success: how optimism undermines executives' decisions. Harvard Business Review, 56-63.

March, J. G. (1994). Primer on Decision Making: How Decisions Happen. New York: NY: Free Press.

McCormick, S. (2006). The Brazilian anti-dam movement: knowledge, contestation as communicative action. Organizational \& Environment, 19(3), 321-346.

Mieg, H. (2009). Two factors of expertise? Excellence and professionalism of environmental experts. High Ability Studies, 20, 91-115.

Mieg, H. A. (2014). The organisational embedding of expertise: Centres of excellence. Talent Development and Excellence, 6, 71-93.

Mintzberg, H., Raisinghani, D., \& Théorêt, A. (1976). The Structure of 'Unstructured' Decision Processes. Administrative Sciences Quarterly, 21, 246-275.

Moritz, L., \& Gieri, H. (September de 2015). Heuristics in organizations: A review and a research agenda. Journal of Business Research, 68 (9), 2027-2036.

Neumann, J. v., \& Morgenstern, O. (1944). Theory of Games and Economic Behavior. Princeton: Princeton University Press.

NU. CEPAL. (2015). The United Nations regional commissions and the 2030 agenda for sustainable development: Moving to deliver on a transformative and ambitious agenda. Santiago de Chile: ECLAC.

Odum, H. (1996). Environmental Accounting: Emergy and Environmental Decision Making. New York: John Wiley and Sons.

Plous, S. (1993). The availibility heuristic. The psychology of judgment and decision making. New York: McGraw-Hill.

Rahim, M. A. (2002). Toward a theory of managing organizational conflict. The International Journal of Conflict Management, 13, 206-235.

Rosso, M., Bottero, M., Pomarico, S., Ferlita, S. L., \& Comino, E. (April de 2014). Integrating multicriteria evaluation and stakeholders analysis for assessing hydropower projects. Energy Policy, 67, 870-881.

Rubenstein, A. (2013). Response time and decision making: An experimental study. Judgment and Decision Making, 8, 540-551.

Shackle, G. (1972). Epistemics \& Economics: A Critique of Economic Doctrines. Cambridge, United Kingdom: Cambridge University Press.

Shmelev, S. E. (2012). Ecological Economics: Sustainability in Practice. Oxford, UK: Springer.

Simon, H. A. (1976). Administrative Behavior: A Study of Decision-Making Processes in Administrative Organization (Third ed.). London: Collier Macmillan Publishers.

Sovacool, B., \& Cooper, C. (2013). The Governance of Energy Megaprojects: Politics, Hubris, and Energy Security. Cheltenham, UK: Edward Elgar Publishing, Incorporated.

Sovacool, B., S.E., R., P.C., S., Janda, K., Rochlin, G., Spreng, D., y otros. (March de 2015). Integrating social science in energy research. Energy Research \& Social Science, 6, 95-99. 
Stone, R. (2011). Hydropower. The legacy of the Three Gorges Dam.. Science, 333, 817.

Thaler, R. H., \& Benartz, S. (2004). Save More Tomorrow: Using Behavioral Economics to Increase Employee Saving. Journal of Political Economy, 112, 164-187.

Tversky, A., \& Kahneman, D. (1974). Judgment under uncertainty: Heuristics and biases. Science(185), 1124-1131.

Tversky, A., \& Kahneman, D. (1986). Rational Choice and the Framing of Decisions. The Journal of Business, 251-278.

Tversky, A., \& Kahneman, D. (1992). Advances in prospect theory Cumulative representation of uncertainty. Journal of Risk and Uncertainty, 5, 297-323.

UN, EC, IMF, OECD and World Bank. (2014). System of Environmental-Economic Accounting 2012: Central Framework - final System of Environmental-Economic Accounting 2012: Central Frameworkic Accounting. New York: United Nations, European Commission, International Monetary Fund, Organisation for Economic Co-operation and Development and World Bank.
Unidad de Planeación Minero Energética - UPME. (2014). PLAN DE EXPANSION DE REFERENCIA EN GENERACION. En U. d. UPME, Plan de Expansión de Referencia Generación - Transmisión (págs. 188-220). Bogotá Colombia: UPME (eds.).

Vroom, V. H., \& Jago, A. G. (1988). The New Leadership: Managing Participation in Organizations. Englewood Cliffs, NJ: Prentice-Hall.

Vroom, V. H., \& Yetton, P. W. (1973). Leadership and DecisionMaking. Pittsburgh: University of Pittsburgh Press.

Wakker, P. P. (2010). Prospect Theory: For Risk and Ambiguity. Cambridge: Cambridge University Press.

Yaari, M. (1987). The Dual Theory of Choice Under Risk. Econometrica, 55, 95-115.

Yüksel, İ. (2012). Developing a Multi-Criteria Decision Making Model for PESTEL. International Journal of Business and Management, 7 (24), 52 - 66. 